\title{
Achieving Highly Efficient Fluorescent Blue Organic Light- Emitting Diodes Through Optimizing Molecular Structures and Device Configuration
}

\author{
Chang-Gua Zhen, Yan-Feng Dai, Wen-Jin Zeng, Zhun Ma, Zhi-Kuan Chen,* \\ and John Kieffer*
}

Based on the results of first-principles calculations of the electronic properties of blue light-emitting materials, the molecular structures of oligofluorenes are optimized by incorporating electron-withdrawing groups into the molecules to balance hole and electron injection and transport for organic light-emitting diodes (OLEDs). The result is a remarkable improvement in the maximum external quantum efficiency (EQE) of the undoped device from $2.0 \%$ to $4.99 \%$. Further optimization of the device configurations and processing procedures, e.g., by changing the thickness of the emitting layer and through thermal annealing treatments, leads to a very high maximum EQE of $7.40 \%$ for the undoped sky-blue device. Finally, by doping the emitter in a suitable host material, 4,4'-bis(carbazol-9-yl)biphenyl (CBP), at the optimal concentration of $6 \%$, pure blue emission with extremely high maximum EQE of $9.40 \%$ and Commission Internationale de l'Eclairage (CIE) coordinates of $(0.147$, 0.139 ) is achieved.

\section{Introduction}

Organic light-emitting diodes (OLEDs) are promising devices for the next generation of flat panel displays and lighting applications. ${ }^{[1-3]}$ High efficiency is required to compete with other technologies such as liquid-crystal display and fluorescent tubes. The external quantum efficiency (EQE) of OLEDs can be expressed as

$\mathrm{EQE}=\gamma \chi \eta_{\mathrm{pl}} \eta_{\mathrm{oc}}$

where $\gamma$ is the recombination efficiency of injected holes and electrons, $\chi$ is the fraction of excitons that can potentially radiatively decay due to restriction of multiplicity, $\eta_{\mathrm{pl}}$ is the intrinsic photoluminescence (PL) efficiency, and $\eta_{\mathrm{oc}}$ is the light

C.-G. Zhen, Prof. J. Kieffer

Department of Materials Science and Engineering

University of Michigan

Ann Arbor, MI 48109, USA

E-mail: kieffer@umich.edu

Y.-F. Dai, Dr. W.-J. Zeng, Dr. Z. Ma, Dr. Z.-K. Chen Institute of Materials Research and Engineering

Singapore 117602

E-mail: zk-chen@imre.a-star.edu.sg

DOI: $10.1002 / \mathrm{adfm} .201002165$ out-coupling factor. Ideally, $\gamma=1$ only if holes and electrons are fully balanced and completely recombined to form excitons. $\eta_{\text {oc }}$ is about $20 \pm 2 \%$, which is estimated from $\eta_{\mathrm{oc}}=1-\left(1-1 / n^{2}\right)^{1 / 2}$, where $n$ is the refractive index of the organic layers (normally it is $1.7 \pm 0.1$ for organic solids).${ }^{[4]} \chi$ depends on the type of OLEDs, either phosphorescent OLEDs (POLEDs) or fluorescent OLEDs (FOLEDs). The former exploit both triplet and singlet excitons while the latter can only utilize singlet excitons to convert electrical energy to light. ${ }^{[5]}$ According to classical degeneracy statistics, the probability of generating triplet excitons is $75 \%$ while it is $25 \%$ for singlet excitons from the recombination of injected carriers, assuming the formation cross-sections for triplet and singlet excitons are equal. ${ }^{[6]}$ Therefore, $\chi$ for POLEDs is $100 \%$ while it is $25 \%$ for FOLEDs. Hence, the classical upper EQE limit of FOLEDs is about 5\%, whereas that of POLEDs is about $20 \%$. However, there are other advantages that FOLEDs have over POLEDs. First, there are many organic materials that exhibit fluorescence while only very few exhibit phosphorescence around room temperature. ${ }^{[7]}$ Second, the efficiency roll-off at high current density in FOLEDs is less severe than in POLEDs, due to a shorter exciton lifetime and less bimolecular quenching. ${ }^{[8-10]}$

Recent progress in experiments ${ }^{[11,12]}$ and theory ${ }^{[13,14]}$ suggest that singlet generation probability for conjugated systems can be larger than $25 \%$. Therefore, it is possible for the EQE of FOLEDs to exceed the $5 \%$ limit. Actually, to name a few reported occurrences, Wei and Chen have realized sky-blue FOLED with $7.87 \%$ EQE by optimizing emitting molecules so as to inhibit intermolecular $\pi-\pi$ stacking, which would reduce the efficiency of OLEDs. ${ }^{[15]}$ Okumoto et al. enhanced the EQE of their doped green FOLEDs from $4.4 \%$ to $9.8 \%$ by replacing the commonly used electron transporter tris(8-hydroxyquinolinato) aluminium $\left(\mathrm{Ala}_{3}\right)$ with 9,10-bis[4-(6-methylbenzothiazol2-yl)phenyl]anthracene (DBzA). ${ }^{[16]}$ Chen et al. achieved $8 \%$ EQE for undoped green FOLED by systematically optimizing the relative thicknesses of the hole injection layer, hole transporting layer, and electron transporting layer, and by trying various cathodes. ${ }^{[17]}$ Most astonishing is that the efficiency of 
Chen's devices can vary by more than one order of magnitude when changing the thickness of the electron transporting layer in the narrow window between 0 and $30 \mathrm{~nm}$. The significant implication of these examples ${ }^{[18-21]}$ is that it is indeed possible to achieve highly efficient FOLEDs through optimization of the molecular structures and device configuration. In this paper, we report the realization of a pure blue FOLED with EQE of $9.40 \%$ and Commission Internationale de l'Eclairage (CIE) coordinates of $(0.147,0.139)$, which we accomplished by optimizing the molecular structures of emitters, the device design, and the device fabrication process.

\section{Method of Investigation}

\subsection{Conceptual Approach}

The purpose of this investigation was to improve the efficiency of FOLEDs. To this end we used first-principles electronic structure calculations to analyze an existing molecular design, designated as Blu1, which we had previously synthesized and characterized in our laboratory. Insights gained from our calculations led to new design concepts. Through systematic variation of the molecular architecture and targeted placement of the key functional groups, we computationally predicted an optimized molecule, Blu2, which was subsequently synthesized and tested to validate our predictions. This approach allows us to identify trends and fundamental knowledge that can serve as general design criteria for OLEDs with improved performance characteristics. Further optimization was also possible at the device level.

\subsection{Computational Details}

We carried out first-principles density functional theory (DFT) and the time-dependent DFT (TDDFT) calculations to determine the electronic structure and carrier properties of the blue emitting molecules (Figure 1). We used the B3LYP exchangecorrelation functional ${ }^{[22]}$ and $6-31 G(d)$ basis set implemented in the Gaussian 03 package. ${ }^{[23]}$ To predict the performance of the various molecular architectures we explored computationally, several key properties pertaining to energy conversion in OLEDs have been evaluated as follows:

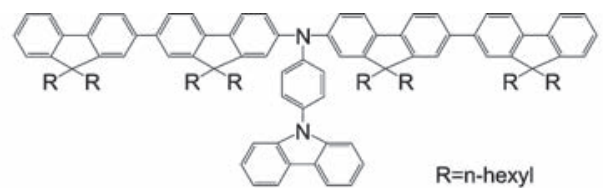

Blu1

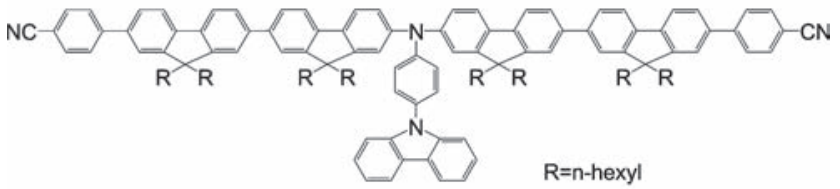

Blu2

Figure 1. Molecular structures of the blue emitters.

\subsubsection{Carrier Injection Barriers}

The hole and electron injection barriers are determined by comparing the work functions of anode and cathode with the energy level of the highest occupied molecular orbital (HOMO) and the lowest unoccupied molecular orbital (LUMO).

\subsubsection{Carrier Transport Properties}

The carrier transport properties are studied in the framework of Marcus' electron transfer model. ${ }^{[24]}$ According to Marcus' model, the conductivity of amorphous organic materials depends on the electron (or hole) transfer reactions between two adjacent molecules (hopping sites), represented by $\mathrm{M}_{1}$ and $\mathrm{M}_{2}$

$\mathrm{M}_{1}^{+/-}+\mathrm{M}_{2} \rightarrow \mathrm{M}_{1}+\mathrm{M}_{2}^{+/-}$.

The corresponding hopping rate can be described by Marcus' equation

$k_{\mathrm{et}}=\frac{2 \pi}{\hbar} \frac{H_{\mathrm{da}}^{2}}{\sqrt{4 \pi \lambda k T}} \exp \left(-\frac{(\Delta G+\lambda)^{2}}{4 \lambda k T}\right)$,

where $\lambda$ is the reorganization energy, $H_{\mathrm{da}}$ the charge-transfer integral, $\Delta G$ the free energy change for the electron transfer reaction, and $T$ the temperature. In the case that the hopping process occurs between identical molecules, $\Delta G$ is zero. According to Equation (3), there are two important parameters dominating the charge transfer process in this model: the charge transfer integral and the reorganization energy. The former originates from the overlap of wavefunction between the hopping sites, which is determined by the relative positions and the spatial wavefunction patterns of the molecules. In amorphous organic solids, $H_{\mathrm{da}}$ can be expected to constitute a less variable quantity due to the random packing of molecules. The other important parameter, the reorganization energy, represents the activation barrier from the configuration adjustment of molecules during the charge transfer. The reorganization energy $\lambda$ consists of inner reorganization energy and external polarization due to the solvent effects of the surrounding medium. Recent studies ${ }^{[25,26]}$ have shown that the inner reorganization energy constitutes the dominant contribution to the charge transfer process in organic materials. Hence, here we focus on inner reorganization energy. The inner reorganization energies are obtained by comparing the energies in charged and uncharged optimized configurations, for both neutral and ionized states, i.e.,

$\lambda=E_{ \pm}^{0}-E_{ \pm}^{ \pm}+E_{0}^{ \pm}-E_{0}^{0}$

where $E_{ \pm}^{0}$ is the energy of the ion in the optimized uncharged geometry, $E_{ \pm}^{ \pm}$is the energy of the ion in the optimized charged geometry, $E_{0}^{ \pm}$is the energy of the neutral molecule in the optimized charged geometry, and $E_{0}^{0}$ is the energy of the neutral molecule in the optimized uncharged geometry.

\subsubsection{Singlet Generation Fraction}

$\chi_{\mathrm{s}}$ of FOLEDs, also referred as singlet generation fraction, is calculated from Shuai's method:[27] 
$\chi_{\mathrm{S}}=\sigma_{\mathrm{S}} /\left(\sigma_{\mathrm{S}}+3 \sigma_{\mathrm{T}}\right)$

$\frac{\sigma_{\mathrm{S}}}{\sigma_{\mathrm{T}}}=\frac{E_{\mathrm{bT}}}{E_{\mathrm{bS}}}$

where $\sigma_{\mathrm{S}}$ and $\sigma_{\mathrm{T}}$ represent the formation cross sections of singlet and triplet excitons, $E_{\mathrm{bS}}$ and $E_{\mathrm{bT}}$ are the binding energies of the singlet and triplet excitons, respectively. $E_{\mathrm{bs}}$ and $E_{\mathrm{bT}}$ are calculated from DFT and TDDFT as ${ }^{[28]}$

$$
\begin{aligned}
& E_{\mathrm{bS}}=E_{\mathrm{g}}-E_{\mathrm{S} 1} \\
& E_{\mathrm{bT}}=E_{\mathrm{g}}-E_{\mathrm{T} 1}
\end{aligned}
$$

where $E_{\mathrm{g}}$ is the HOMO-LUMO energy gap, while $E_{\mathrm{S} 1}$ and $E_{\mathrm{T} 1}$ are the excitation energies from the ground state to the lowest excited singlet state and the lowest excited triplet state, respectively. To reduce the computational time, hexyl was replaced by methyl group in the oligomers, since this does not affect the optical and electronic properties much. ${ }^{[29]}$

\section{Results and Discussion}

We compare the performance of devices based on two different molecular architectures, labeled Blu1 and Blu2, of which the second results from simulation-based design optimization. The underlying molecular structures are shown in Figure 1, and the key device characteristics of the two systems are summarized in Table 1.

The starting point, Blu1, consists of an electron-donating group, 4-carbazolephenyl incorporated into the backbone of the oligofluorene to facilitate hole injection and thereby reduce the operating voltage. ${ }^{[30]}$ We fabricated an undoped device using Blu1 as emitting layer (EML) with the configuration: ITO/PEDOT:PSS/Blu1 (70 nm)/TPBI/LiF/Al (device 1). The turn on voltage, at which the brightness reaches
$1 \mathrm{~cd} \mathrm{~m} \mathrm{~m}^{-2}$, is $3.4 \mathrm{~V}$, which is acceptable. However, its EQE is not satisfactory. The maximum EQE is only $2.0 \%$ at $4.57 \mathrm{~mA} \mathrm{~cm}^{-2}\left(84 \mathrm{~cd} \mathrm{~m}^{-2}\right)$ and drops to $0.53 \%$ at $113 \mathrm{~mA} \mathrm{~cm}^{-2}$ $\left(554 \mathrm{~cd} \mathrm{~m}^{-2}\right)$.

To understand the reason for the low EQE, we carried out first-principles calculations of Blu1 in vacuum. The LUMO is calculated to be $-1.36 \mathrm{eV}$ while the $\mathrm{HOMO}$ is $-4.80 \mathrm{eV}$. The work function of the device anode (ITO/PEDOT:PSS) is about $4.8 \mathrm{eV}$. Hence, the injection barrier for holes is expected to be small. The work function of the device cathode (Al) is about $4.1 \mathrm{eV}$, which creates a high barrier for electron injection even though we use LiF and TPBI to aid electron injection. We also calculate the reorganization energy associated with the charge transport barriers for hole and electron hopping between Blu1 molecules. The reorganization energy for hole hopping, $\lambda_{+}$, is $0.172 \mathrm{eV}$ while the one for electron hopping, $\lambda_{-}$, is $0.232 \mathrm{eV}$. The higher barriers for injection and transport of electrons compared to those for holes leads to a low balance factor $\gamma$, which reduces the EQE. To solve this problem and make injection and transport of electrons easier, we modified the molecular structure of Blu1 by adding electron-withdrawing groups, 4-cyanophenyl, constructing Blu2.

Using the same calculation approach as above, we determined the LUMO of the new molecule to be $-1.86 \mathrm{eV}$ in vacuum, which is $0.50 \mathrm{eV}$ lower than that of Blu1. Experimentally, the LUMO for Blu1 and Blu2 are $-2.12 \mathrm{eV}$ and $-2.31 \mathrm{eV}$ respectively, as determined from the onset voltage of cyclic voltammetry (CV) measurements and optical gaps. Thus, the calculation results are in qualitative agreement with the experimental results. The lower LUMO is expected to lower the electron injection barrier. The reorganization energies of Blu2 for hole hopping and electron hopping are $0.147 \mathrm{eV}$ and $0.183 \mathrm{eV}$, respectively. Both of $\lambda_{+}$and $\lambda_{-}$are smaller than those for Blu1, indicating that Blu2 may be a better carrier transporter than Blu1. More importantly, the difference between $\lambda_{+}$and $\lambda_{-}$for Blu2 is smaller than for Blu1, which is expected to lead to a more balanced carrier transport.

\begin{tabular}{|c|c|c|c|c|c|c|}
\hline Device & EML & Turn on Voltage [V] & $\begin{array}{l}\text { Maximum Current Efficiency }\left[\mathrm{cd} \mathrm{A}^{-1}\right] \\
\text { and EQE [\%] @ J }\left[\mathrm{mA} \mathrm{cm}^{-2}\right]^{\mathrm{a})}\end{array}$ & $\begin{array}{l}\text { Current Efficiency }\left[\mathrm{cd} \mathrm{A}^{-1}\right] \text { and } \\
\text { EQE (\%)@ @ }\left[\mathrm{mA} \mathrm{cm}^{-2}\right]^{\mathrm{a})}\end{array}$ & $\begin{array}{c}\text { Power Efficiency } \\
{\left[\mathrm{lm} \mathrm{W} \mathrm{W}^{-1}\right] @ 100 \mathrm{~cd} \mathrm{~m}^{-2}}\end{array}$ & $\operatorname{CIE}(x, y)$ \\
\hline 1 & Blut (70 nm) & 3.4 & 1.83, $2.00 @ 4.57$ & $0.49,0.53 @ 113$ & 0.89 & $(0.151,0.097)$ \\
\hline 2 & Blu2(87 nm) & 3.1 & 9.51,4.99@16.3 & 7.62,4.00@187 & 4.82 & $(0.161,0.314)$ \\
\hline 3 & Blu2(39 nm) & 2.9 & 10.76, $5.80 @ 7.56$ & 8.50,4.58@99.3 & 6.42 & $(0.159,0.303)$ \\
\hline 4 & Blu2 $(60 \mathrm{~nm})$ & 3.0 & 11.16,6.12@4.44 & 8,78,4.81@113 & 7.23 & $(0.148,0.280)$ \\
\hline 5 & Blu2(100 nm) & 3.4 & 6,80,3.85@14.4 & 6,33,3.58@95.7 & 3.65 & $(0.144,0.279)$ \\
\hline 6 & Blu2 $(60 \mathrm{~nm})$ Annealed & 3.1 & 13.27, $7.40 @ 6.39$ & $9.24,5.16 @ 103$ & 8.55 & $(0.149,0.292)$ \\
\hline 7 & PVK:2\% Blu2 (60 nm) & 5.5 & 2.64,2.71@0.04 & 1.29,1.33@95.1 & 0.70 & $(0.147,0.135)$ \\
\hline 8 & CBP:2\% Blu2 (60 nm) & 3.9 & 6.40,6.88@1.00 & 4.20,4.51@104 & 3.05 & $(0.147,0.119)$ \\
\hline 9 & CBP:4\% Blu2 $(60 \mathrm{~nm})$ & 4.0 & 8.77,8.60@1.23 & 5.67, $5.56 @ 109$ & 4.28 & $(0.149,0.132)$ \\
\hline 10 & CBP:6\% Blu2 (60 nm) & 3.9 & 10.08, $9.40 @ 0.69$ & 5.48,5.11@110 & 4.29 & $(0.147,0.139)$ \\
\hline 11 & CBP:8\% Blu2 $(60 \mathrm{~nm})$ & 4.2 & 9.11,8.07@1.04 & 6.27, $5.55 @ 99.9$ & 4.34 & $(0.147,0.149)$ \\
\hline 12 & CBP:10\% Blu2 $(60 \mathrm{~nm})$ & 3.9 & 8,88, 7.26@0.88 & 6,30,5.15@96.5 & 4.51 & $(0.149,0.166)$ \\
\hline
\end{tabular}

Table 1. Summary of device performances of ITO/PEDOT:PSS $(50 \mathrm{~nm}) / \mathrm{EML} / \mathrm{TPBI}(30 \mathrm{~nm}) / \mathrm{LiF}(0.5 \mathrm{~nm}) / \operatorname{Al}(150 \mathrm{~nm})$.

a) The first number is current efficiency, the second number is $E Q E$, the third number is J, current density. 
To test our prediction of the charge transport properties of Blu1 and Blu2, we fabricated hole-only and electron-only devices to obtain hole and electron mobility by using the space-charge limited current (SCLC) method ${ }^{[31]}$ with device configurations of ITO/Blu1 or Blu2 (100 nm)/Ag and ITO/Ca(20 nm)/Blu1 or Blu2 $(100 \mathrm{~nm}) / \mathrm{Ca}(20 \mathrm{~nm}) / \mathrm{Ag}$, for hole-only and electron-only devices, respectively. The mobilities were determined by fitting the current to the model of a single carrier SCLC, which is described as

$J=\frac{9}{8} \varepsilon_{0} \varepsilon_{\mathrm{r}} \mu_{0} \frac{V^{2}}{L^{3}}$

where $J$ is the current density, $\mu_{0}$ is the zero-field mobility, $\varepsilon_{0}$ is the permittivity of free space, $\varepsilon_{\mathrm{r}}$ is the relative permittivity of the material (assumed to be 3 for organic materials), $L$ is the thickness of the organic layer, and $V$ is the applied voltage. The $J-V^{2}$ plots for the devices are shown in Figure 2. Hole mobilities of Blu2 and Blu1 are found to be $5.3 \times 10^{-5}$ and $4.1 \times 10^{-5} \mathrm{~cm}^{2} \mathrm{~V}^{-1} \mathrm{~s}^{-1}$. Amazingly, we find the electron mobility of Blu2 to be
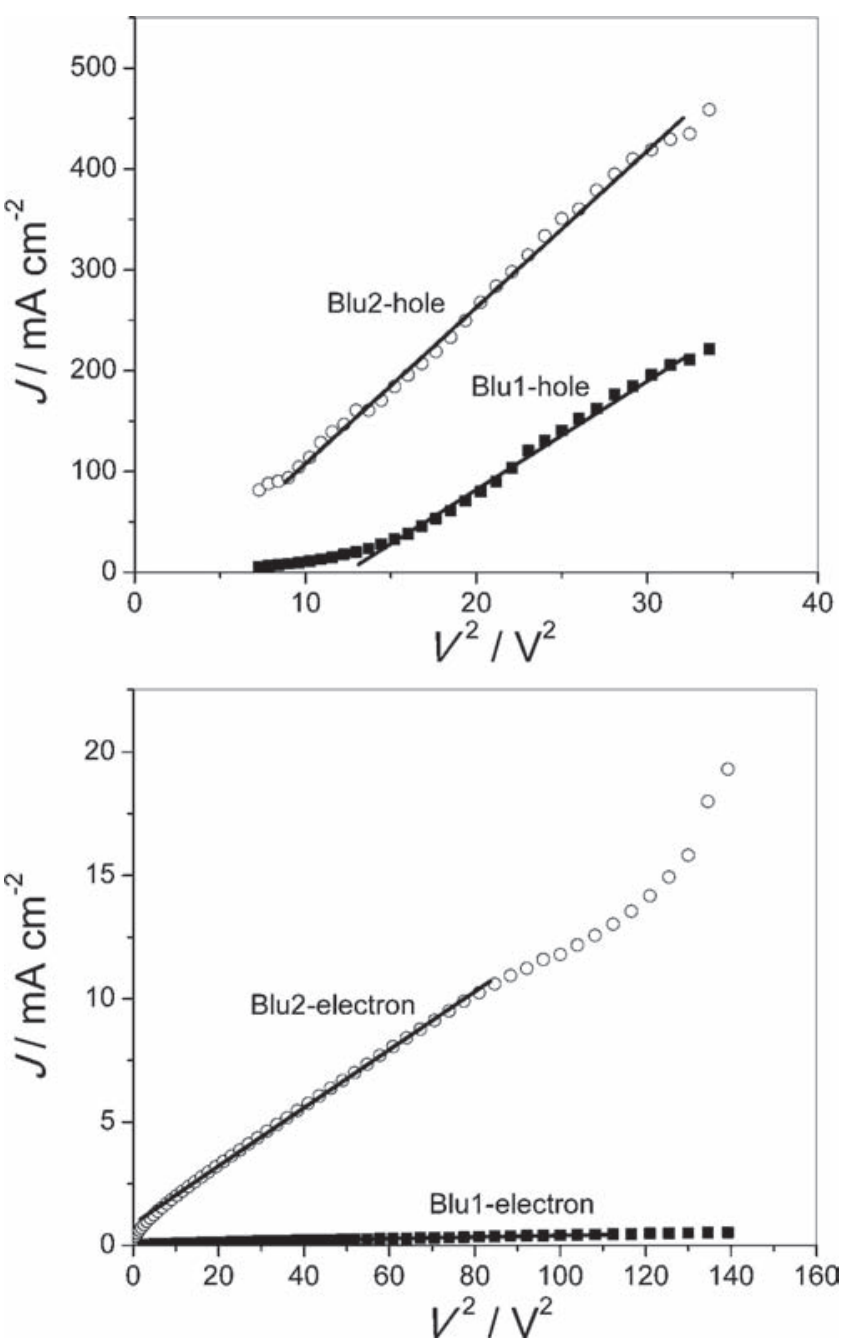

Figure 2. $J-V^{2}$ characteristics of devices of ITO/Blu1 or Blu2 $(100 \mathrm{~nm}) /$ $\mathrm{Ag}$ (top) and ITO/Ca(20 nm)/Blu1 or Blu2 $(100 \mathrm{~nm}) / \mathrm{Ca}(20 \mathrm{~nm}) / \mathrm{Ag}$ (bottom). The solid lines represent best fits to Equation (9).
$4.0 \times 10^{-7} \mathrm{~cm}^{2} \mathrm{~V}^{-1} \mathrm{~s}^{-1}$, 36 times larger than the $1.1 \times 10^{-8} \mathrm{~cm}^{2} \mathrm{~V}^{-1} \mathrm{~s}^{-1}$ of Blu1, confirming the importance of the reorganization energies in controlling charge transport, as discussed above.

Comparing our computational and experimental results above for Blu2 and Blu1, we anticipate a higher balance factor for device 2, which contains a $87 \mathrm{~nm}$ thin film of Blu2 as emitting layer in a configuration similar to that of device 1 .

As expected, the performances of device 2 are much better than device 1 . Not only is the EQE of device 2 higher, but also the efficiency roll-off is improved with respect to device 1 (Figure 3). Device 2 shows a maximum EQE of $4.99 \%$ at $16.3 \mathrm{~mA} \mathrm{~cm}$ (1552 $\mathrm{cd} \mathrm{m} \mathrm{m}^{-2}$ ). The efficiency of device 1 began to drop off severely at about $10 \mathrm{~mA} \mathrm{~cm}{ }^{-2}$. By contrast, even at high current density of $187 \mathrm{~mA} \mathrm{~cm}{ }^{-2}\left(14243 \mathrm{~cd} \mathrm{~m}^{-2}\right)$, the EQE of device 2 is still as high as $4.00 \%$. The efficiency roll-off at high current density originates from exciton quenching or unbalance of carriers in the emitting layer. ${ }^{[8]}$ The better efficiency roll-off of device 2 at high current density indicates that quenching of excitons is small and/or balanced charge injection and transport in the emitting layer of Blu2 prevail even at high electric field. Therefore, the optimized molecular structure of the emitters leads to higher EQE and better device performance at high current density.

Further improvement of the device performance could be achieved by varying thickness of Blu2. We fabricated devices with $39 \mathrm{~nm}$ (device 3), $60 \mathrm{~nm}$ (device 4), and $100 \mathrm{~nm}$ (device 5) EML thickness. The quantum efficiency strongly depends on the thickness (Figure 4). The best performance comes from device 4 with a maximum EQE of $6.12 \%$ at $4.44 \mathrm{~mA} \mathrm{~cm}^{-2}\left(496 \mathrm{~cd} \mathrm{~m}^{-2}\right)$ and drops to $4.81 \%$ at high current density of $113 \mathrm{~mA} \mathrm{~cm}^{-2}\left(9950 \mathrm{~cd} \mathrm{~m}^{-2}\right)$. The thickness dependence of the EQE either arises from microcavity effects or carrier diffusion length inside the emitting layer. ${ }^{[32]}$ In case of the former reason, there should be a threshold current for light emission, because emission would be stimulated rather than spontaneous. ${ }^{[3]}$ In the case of our devices, as a representative example, the EL intensity of device 4 is linear with current density over more than three orders of magnitude and can be extrapolated to the origin (Figure 5). We do not observe such a threshold, indicating that the microcavity effect is not important for the dependence of EQE on thickness. Therefore, it is more plausible that the recombination efficiency of carriers varies in EML of different thickness, due to the variation of diffusion lengths of carriers inside the EML. The dependence of EQE on thickness indicates that the overlap region where electrons and holes can recombine efficiently is about $60 \mathrm{~nm}$ in thickness. For thinner EML, a significant fraction of injected holes diffuse out of the EML without recombining with electrons, thus reducing the recombination efficiency and the overall EQE. For thicker emitting layer, part of holes will be trapped in the EML far from the electron rich region and undergo non-radiative recombination. ${ }^{[32]}$ A similar analysis applies to electrons injected from electron transport layer.

The stability of Blu2 is also very encouraging. For comparison, device 6 with $60 \mathrm{~nm}$ spin-coated Blu2 layers was annealed at $120^{\circ} \mathrm{C}\left(T_{\mathrm{g}}=101.7^{\circ} \mathrm{C}\right)$ in air for $2 \mathrm{~h}$ before depositing TPBI. The EL spectrum of the annealed device is almost the same as the unannealed one (Figure 6), indicating the emitting material is very stable to oxidation and attack by moisture, which is important to prevent degradation in applications demanding long-term operation. Actually, the device efficiency is even higher with the thermal aging treatment compared with device 4 , which 


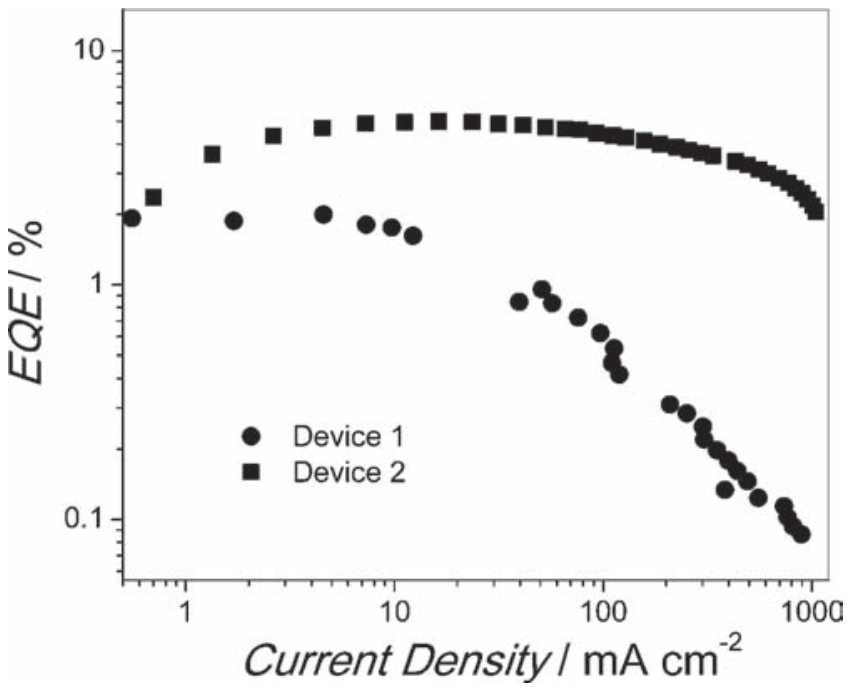

Figure 3. Comparison of the EQE of device 1 (ITO/PEDOT:PSS $(50 \mathrm{~nm}) /$ $\operatorname{Blul}(70 \mathrm{~nm}) / \mathrm{TPBI}(30 \mathrm{~nm}) / \mathrm{LiF}(0.5 \mathrm{~nm}) / \mathrm{Al}(150 \mathrm{~nm}))$ and device 2 (ITO/ PEDOT:PSS(50 nm)/Blu2(87 nm)/TPBI $(30 \mathrm{~nm}) / \mathrm{LiF}(0.5 \mathrm{~nm}) / \mathrm{Al}(150$ $\mathrm{nm})$ ) as a function of current density.

contains an unannealed EML. The maximum EQE of device 6 is $7.40 \%$ at $6.39 \mathrm{~mA} \mathrm{~cm}^{-2}\left(848 \mathrm{~cd} \mathrm{~m}^{-2}\right)$ and drops to $5.16 \%$ at $103 \mathrm{~mA} \mathrm{~cm}^{-2}$ (9493 $\left.\mathrm{cd} \mathrm{m}^{-2}\right)$. A possible reason for the better performance of device 6 is that the annealing reduces the morphology defects such as pinholes and protrusions in the emitting layer, as uniformity of the organic layers is essential for OLEDs. ${ }^{[34]}$ Indeed, the surface morphology study using atomic force microscopy (AFM) of the annealed and unannealed EML before deposition of TPBI reveals that the annealing treatment can improve the uniformity of the films (Figure 7), reducing the surface roughness amplitude from $6.79 \mathrm{~nm}$ to $3.11 \mathrm{~nm}$.

Although the efficiency of device 6 is quite high, the color of the emission is sky-blue with CIE coordinates (0.149, 0.292), which falls out of the range of pure blue color with CIE

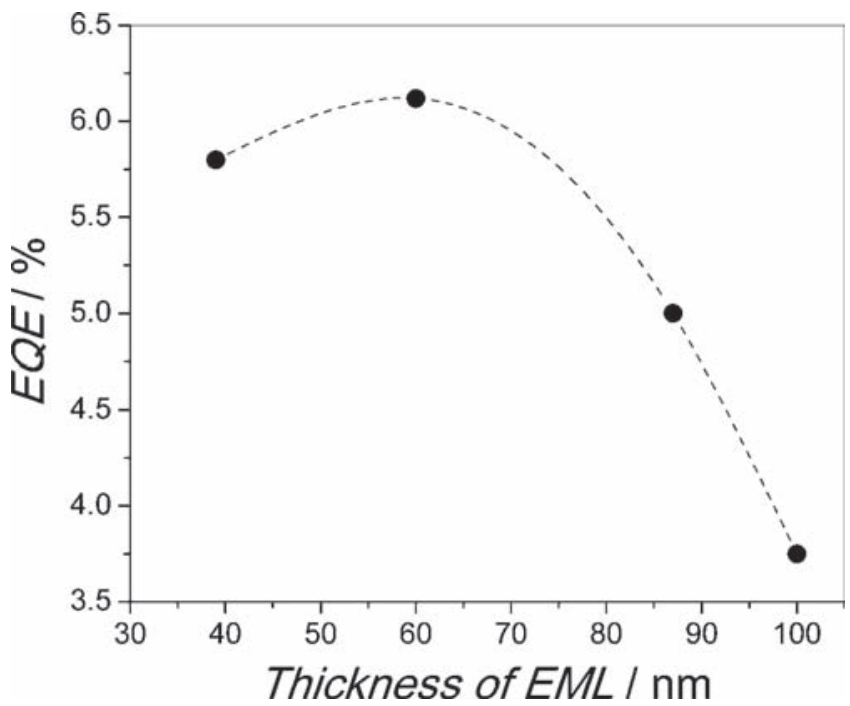

Figure 4. The correlation between EQE and thickness of undoped devices using Blu2 as emitting layer.

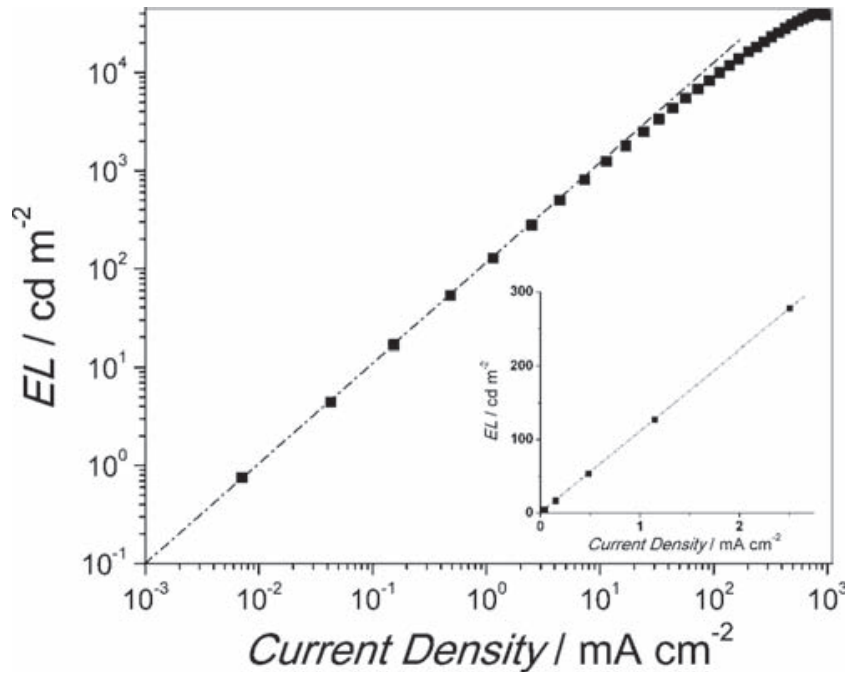

Figure 5. Dependence of output intensity of device 4 (ITO/ PEDOT:PSS(50 nm)/Blu2(60 nm)/TPBI (30nm)/LiF(0.5 nm)/Al(150 nm)) on the injection current density. The insertion shows that the linearity relationship can be extrapolated to the origin.

$x+y<0.30$. The emission peak is at $493 \mathrm{~nm}$ and is significantly red-shifted compared to the PL emission of the molecule in dilute toluene solution at concentration of $2 \times 10^{-6} \mathrm{~mol} \mathrm{~L}^{-1}$, which is at $437 \mathrm{~nm}$. The difference between PL emission from a dilute solution and EL emission from the solid state under an electric field implies that the interaction between emitting molecules in the solid state is strong. To further improve the performance of the device, especially to bring its color into the pure blue range, we created EML materials that consist of $2 \%$ $(\mathrm{w} / \mathrm{w})$ Blu2 doped into an appropriate host material, designed to reduce the interactions between the emitting molecules, while preserving the overall configuration of device 6 . We tested two materials as host: polymeric PVK (poly(9-vinyl carbazole)) for device 7 and small molecular CBP (4,4'-bis(carbazol-9-yl)

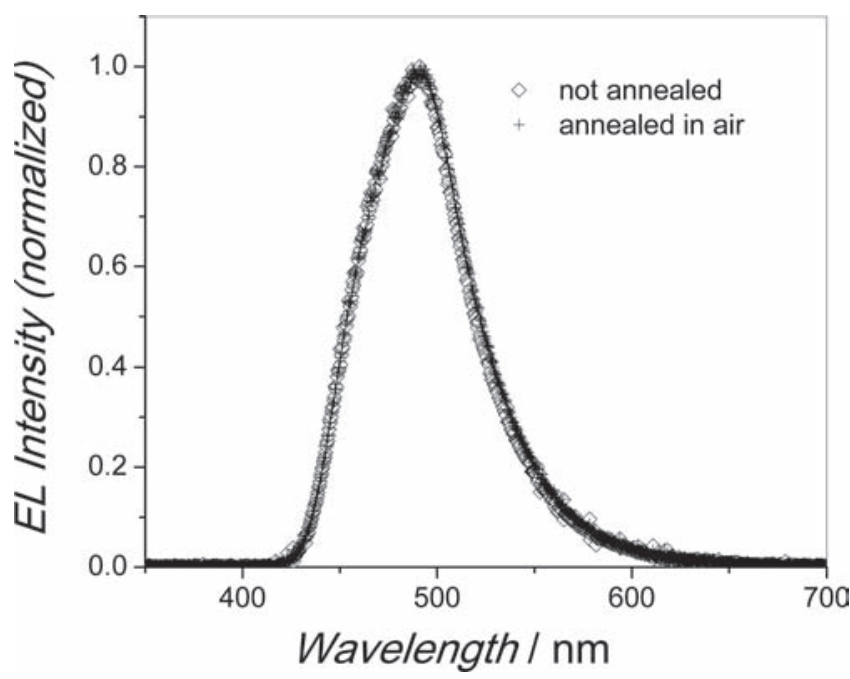

Figure 6. The EL spectrums of device 4 and device 6 comprising EML unannealed and annealed in air at $120^{\circ} \mathrm{C}$ for $2 \mathrm{~h}$. 


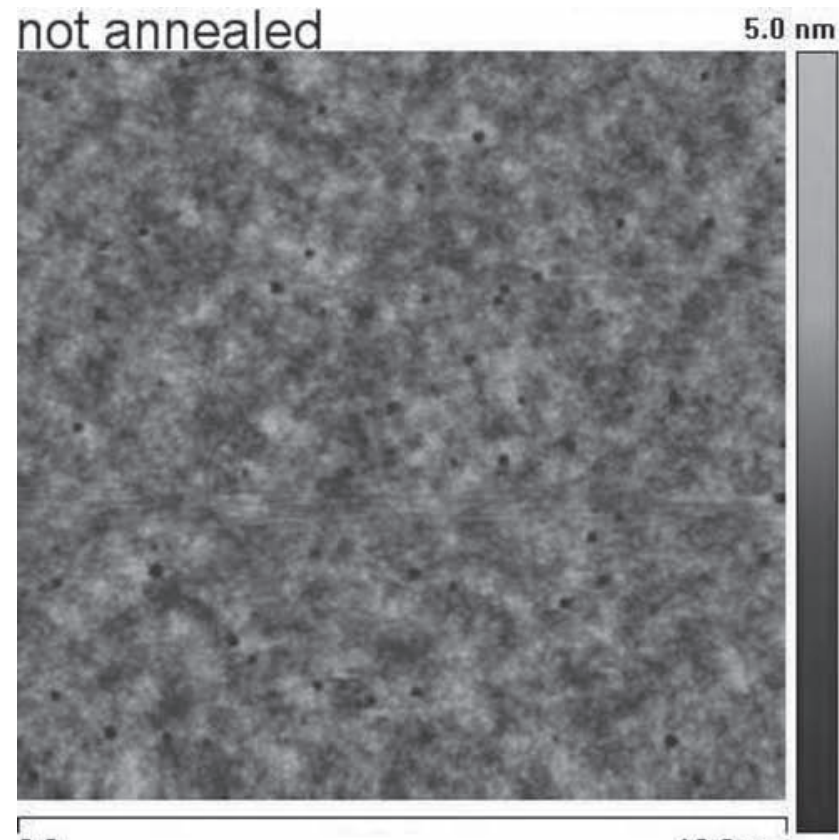

0.0

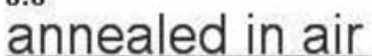

$10.0 \mu \mathrm{m}$

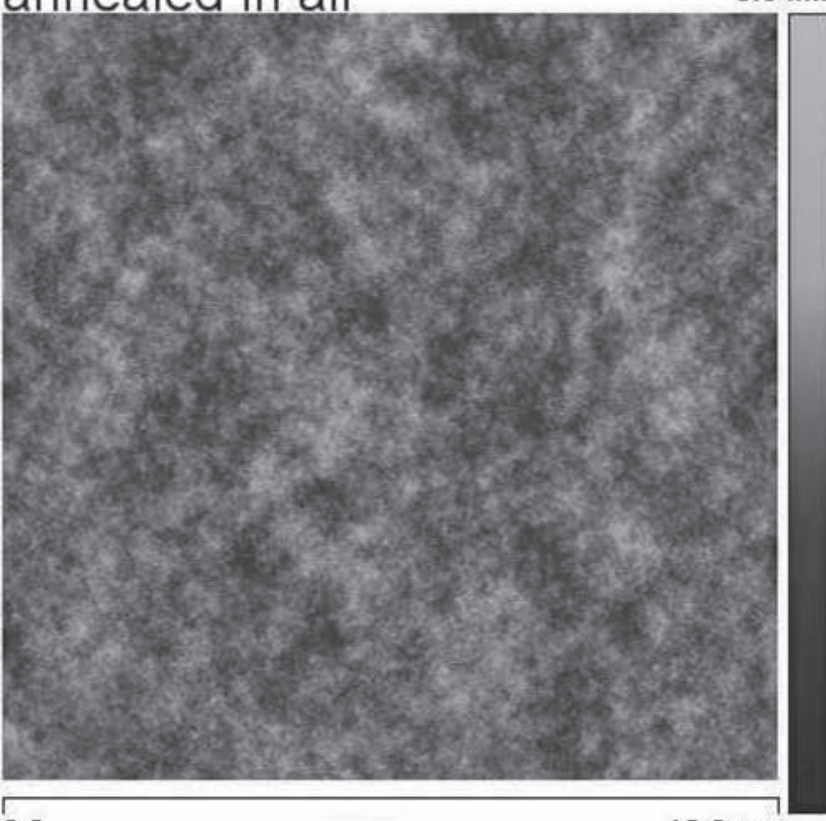

0.0

$10.0 \mu \mathrm{m}$

Figure 7. The surface morphology of EML before deposition of TPBI. Top: unannealed EML with a surface roughness amplitude of $6.79 \mathrm{~nm}$. Bottom: EML annealed in air at $120^{\circ} \mathrm{C}$ for $2 \mathrm{~h}$ with a surface roughness amplitude of $3.11 \mathrm{~nm}$.

biphenyl) for device 8 . There is good overlap between the PL spectra of the chosen hosts and the absorption of the dopant (Figure 8). Therefore, the Förster energy transfer from host to dopant is expected to be efficient.

The emission from device 7 is in the range of pure blue with CIE coordinate $(0.147,0.135)$, and the maximum EQE is $2.71 \%$. The turn on voltage is $5.5 \mathrm{~V}$, much higher than device 6 using non-doped Blu2 as emitting layer. Compared to device 7, the

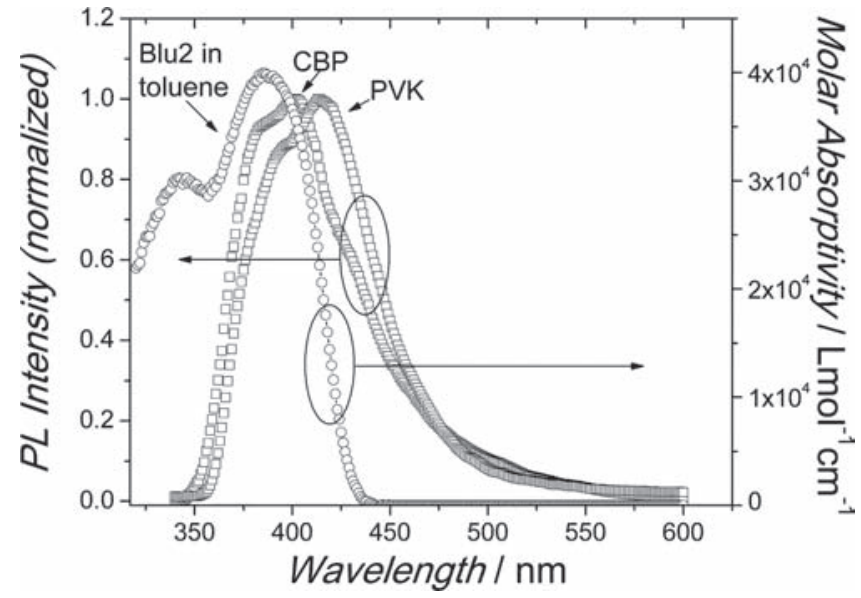

Figure 8. The absorption spectrum of Blu2 in toluene and photoluminescence spectra of CBP and PVK films. CBP film was thermally deposited and PVK film was spin-coated from ethyl benzoate.

performance of device 8 is much better. The CIE coordinates of the emission are $(0.147,0.119)$, within the range of pure blue. The maximum EQE is $6.88 \%$ at $1.0 \mathrm{~mA} \mathrm{~cm}^{-2}\left(64 \mathrm{~cd} \mathrm{~m}^{-2}\right)$ and drops to $4.51 \%$ at $104 \mathrm{~mA} \mathrm{~cm}^{-2}\left(4389 \mathrm{~cd} \mathrm{~m}^{-2}\right)$. The poor performance of the device using PVK as host material compared the one using CBP may have several reasons. The first one comes from the spectral overlap integral $(J)$, which determines the efficiency of energy transfer from host to dopant. It can be calculated as ${ }^{[35]}$

$J=\frac{\int_{0}^{\infty} \lambda^{4} f_{\mathrm{D}}(\lambda) \varepsilon_{\mathrm{a}}(\lambda) \mathrm{d} \lambda}{\int_{0}^{\infty} f_{\mathrm{D}}(\lambda) \mathrm{d} \lambda}$

where $f_{\mathrm{D}}$ is the normalized PL spectrum of donors (PVK or $\mathrm{CBP}$ ) and $\varepsilon_{\mathrm{a}}$ is the molar extinction coefficient of the acceptor (Blu2). The calculated spectral overlap integral between CBP and Blu2 is $5.15 \times 10^{14} \mathrm{~L} \mathrm{~mol}^{-1} \mathrm{~cm}^{-1} \mathrm{~nm}^{-4}, 11.4 \%$ larger compared to that between PVK and Blu2, which is $4.63 \times$ $10^{14} \mathrm{~L} \mathrm{~mol}^{-1} \mathrm{~cm}^{-1} \mathrm{~nm}^{-4}$. The second reason is the poor electron injection and transport capability of PVK due to its low electron affinity (EA) and its poor electron mobility. The third reason is that the exciton lifetime of PVK is orders of magnitude shorter than that of CBP. ${ }^{[36]}$ The excitons generated in the PVK host can not completely transfer to the dopant molecules before they decay to their ground state.

The efficiency of doped device 8 is a little lower than that of undoped device 6 . This is probably due to the incomplete transfer of the excitation energy from the host to the dopant. To find ways for improving the efficiency of the doped devices, we investigated the energy transfer efficiency as a function of doping concentration of Blu2 in CBP. Based on Förster's formulation, the critical distance $R_{0}$ at which $50 \%$ of the excitation energy is transferred from the donor to acceptor is

$R_{0}=\left(\frac{(9 \ln 10) K^{2} Q_{\mathrm{a}} J}{128 \pi^{2} n^{4} N_{\mathrm{A}}}\right)^{1 / 6}$

where $\kappa^{2}$ is the orientation factor determined by the angle between donor and acceptor dipoles, $Q_{\mathrm{d}}$ the PL efficiency of 
the donor in the absence of acceptor, $J$ spectral overlap integral, $n$ the refractive index of the medium containing donor and acceptor, and $N_{\mathrm{A}}$ Avogadro's number. In our host-dopant system, $\kappa^{2}$ equals to $2 / 3$ assuming the host and dopant molecules are oriented randomly, $Q_{\mathrm{d}}$ is 0.61 for $\mathrm{CBP},{ }^{[37]} \mathrm{J}$ is calculated as reported above, $n$ is about 1.8 for CBP. ${ }^{[38]}$ We obtain $R_{0}=34.8 \AA$. To calculate the critical dopant concentration, $C_{0}$, for which more than $50 \%$ excitation energy transfer is expected from the host material to the dopant molecules, we assume that all dopant molecules are evenly distributed in the host material, effectively occupying the sites of a simple cubic lattice. The length of the space diagonal of the unit cell in this lattice is $2 R_{0}$ and the longest distance between any host molecule and its nearest dopant molecule is $R_{0}$. The critical dopant concentration is

$C_{0}=\frac{m_{\text {dopant }}}{\frac{8 \sqrt{3}}{9} R_{3}^{0} \rho_{\text {host }}}$

where $m_{\text {dopant }}$ is the mass of a dopant molecule, $\rho$ the density of the host material (about $1.3 \mathrm{~g} \mathrm{~cm}^{-3}$ for $\mathrm{CBP}^{[39]}$ ). Using the calculated $R_{0}$, we find $C_{0}=4.6 \%$. For efficient energy transfer from CBP to Blu2, the doping concentration should not be less than $\mathrm{C}_{0}$. We prepared EML layers with doping concentrations of $4 \%$, $6 \%, 8 \%$, and $10 \%$ for device 9 , device 10 , device 11 , and device 12 , respectively. The corresponding CIE coordinates are (0.149, $0.132),(0.147,0.139),(0.147,0.149)$, and $(0.149,0.166)$. All the emissions are in range of, or very close to, pure blue color. The best device performance comes from device 10 with doping concentration of $6 \%$ (Figure 9), which shows the highest maximum EQE of $9.40 \%$ at $0.69 \mathrm{~mA} \mathrm{~cm}^{-2}\left(69 \mathrm{~cd} \mathrm{~m}^{-2}\right)$ and still maintains $5.11 \%$ at $110 \mathrm{~mA} \mathrm{~cm}^{-2}\left(6055 \mathrm{~cd} \mathrm{~m}^{-2}\right)$. As expected, increasing the doping concentration from $2 \%$ to $6 \%$ enhances the EQE of these devices due to a more efficient energy transfer from the host to the dopants. However, further increase of the doping concentration induces a lower EQE. The decline of the EQE may come from non-radiative quenching due to the aggregation

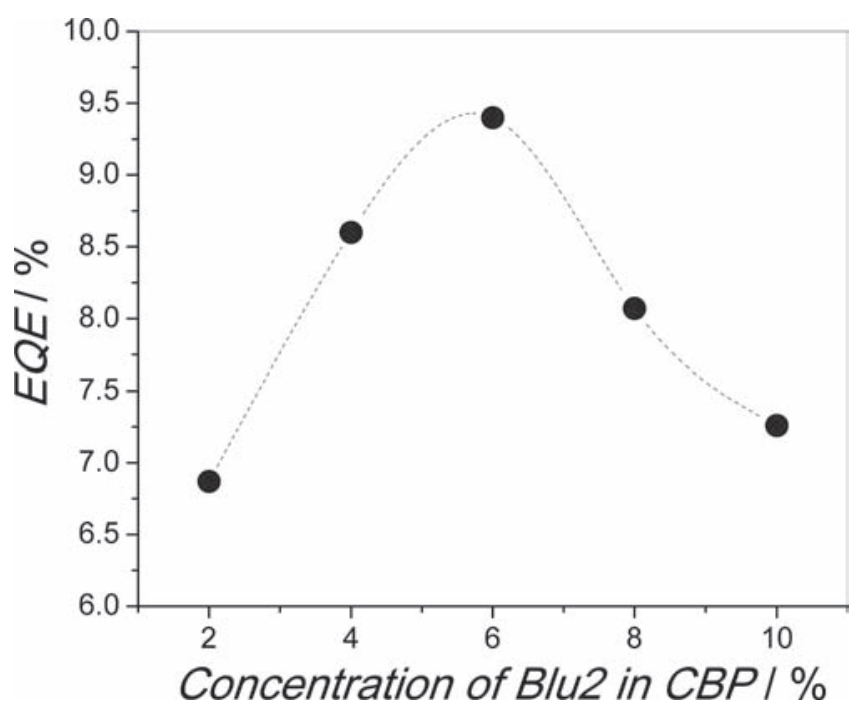

Figure 9. The maximum $\mathrm{EQE}$ as a function of doping concentration of Blu2 in CBP host.

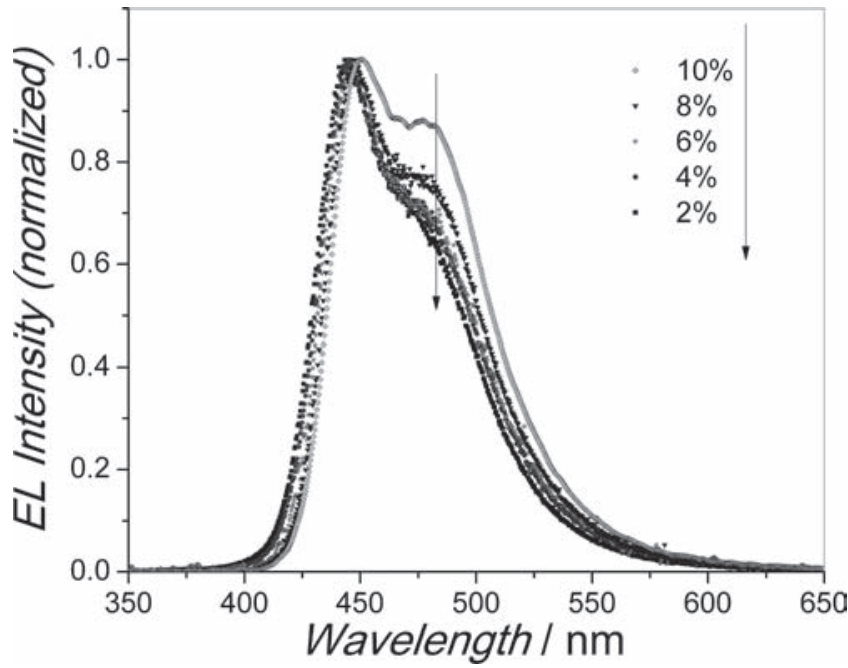

Figure 10. EL spectra of devices with different doping concentration of Blu2 in CBP host.

of dopant molecules at high doping concentrations. ${ }^{[40]}$ Indeed, we observed that the component of the spectra at long wavelength increases significantly as the doping concentration exceeds 6\% (Figure 10), which is a sign of aggregation of the emitting molecules. ${ }^{[41]}$

To understand the origin of the high EQE of our devices, we need to examine the factors in Equation (1) one by one. First, we determined $\eta_{\mathrm{pl}}$ for thin films of pure Blu2 and Blu2 doped in CBP (4\%) host using an integrating sphere. ${ }^{[37]}$ The PL efficiency of these solid thin films is $90 \% \pm 5 \%$ in both cases. The high PL efficiency of the emitting material indicates that the fraction of excitons that decay through non-radiative pathway is negligible.

The other important factor, $\chi$, is the singlet exciton generation fraction in FOLEDs. The singlet exciton can be generated directly from the recombination of injected carriers or from triplet-triplet $(\mathrm{T}-\mathrm{T})$ annihilation: ${ }^{[42]}$

${ }^{3} \mathrm{Blu}^{*}+{ }^{3} \mathrm{Blu}^{*} \rightarrow{ }^{1} \mathrm{Blu}^{*}+$ Blu2,

This process will generate two singlet excitons out of every ten triplet excitons, assuming the generation probability of triplet excitons is $75 \%$ while it is $25 \%$ for singlet excitons from T-T annihilation. ${ }^{[43]}$ If the fraction of singlet excitons generated from triplet-triplet annihilation is non-negligible among the all singlet excitons, the luminance will increase more than linearly with increasing current density. This is because the singlet excitons formation through $\mathrm{T}-\mathrm{T}$ annihilation is second-order with respect to the concentration of ${ }^{3} \mathrm{Blu2}{ }^{*}$, which in turn is linear with the current density. ${ }^{[44]}$ For all our devices, the luminance increases linearly with increasing current density at low current injection and less than linearly at higher current injection, as shown in Figure 5. Therefore, the T-T annihilation does not play a critical role in achieving high EQE for our devices.

According to classical statistics, the singlet exciton fraction generated from direct recombination is $25 \%$, assuming the formation cross-section of singlet and triplet excitons is similar. A recent theoretical study indicates that this assumption may not be correct. ${ }^{[3,45]}$ The formation cross-section ratio of singlet to triplet 
excitons for some oligomers and polymers lies between 2 to 5 , as determined by photoinduced absorption and magnetic resonance experiments ${ }^{[12]}$ and an equation-of-motion coupled cluster theoretical study. ${ }^{[46]}$ Accordingly, the singlet generation fraction is about $40-62.5 \%$. Using Shuai's method, ${ }^{[27]}$ we obtained $46 \%$ of $\chi$ for Blu2 and $42 \%$ for CBP. The exciton formation can be formed either on host molecules or directly on dopant molecules. ${ }^{[2,47]}$ Therefore, for doped devices, $\chi$ is in the range of $42-46 \%$..

Based on the measured PL efficiency $(90 \pm 5 \%)$, the calculated $\chi$ (46\% for undoped and $42-46 \%$ for doped devices), and the estimated out-coupling factor $(20 \pm 2 \%)$, the maximum $\mathrm{EQE}$ is projected to be $8.3 \pm 1.3 \%$ for the undoped device and $8.0 \pm 1.6 \%$ for the doped device, assuming $\gamma$, the recombination efficiency, can reach unity after optimization of device configurations and fabrication process. The highest EQE of the undoped and doped devices after optimization are located in the projected range, indicating that our analysis is reasonable.

\section{Conclusions}

Stable, pure blue light emitting materials with optimized molecular structures have been developed by incorporating both electron donating and electron withdrawing moieties into the molecules to balance hole and electron injection/transporting. Molecular designs are based on the electronic structure and carrier properties of the blue emitting materials obtained from first-principles DFT and the TDDFT calculations. We have achieved EQE as high as 7.4\% and 9.4\% for undoped and doped blue fluorescent OLEDs, respectively, through optimizing the film thickness of active layer and doping concentration (for doped devices). Our results exceed the traditional $5 \%$ limit for fluorescent OLEDs but agree with recent experimental and theoretical studies. Our efforts clearly indicate that fluorescent materials have a bright future for highly efficient and stable OLEDs for flat-panel display and lighting applications.

\section{Experimental Section}

Chemicals: The synthesis of the emitters followed the procedure described in our previous report. ${ }^{[48]}$ Poly(3,4-ethylenedioxythiophene) (PEDOT) doped with poly(styrenesulfonate) (PSS), was purchased from Bayer, Germany. The host material, poly(9-vinyl carbazole) (PVK) $(\mathrm{Mn} \approx 1000000)$, was purchased from Aldrich. Another host material, 4,4'-bis (carbazol-9-yl)biphenyl (CBP), and an electron transport and hole blocking material, 1,3,5-tris (phenyl-2-benzimidazolyl)benzene (TPBI), were purchased from Lumtec Corp., Taiwan. All these materials were used without further purification for device fabrication.

Instruments: The absorption spectra were recorded using a Shimadzu UV-3101PC UV-vis-NIR scanning spectrophotometer. The photoluminescent spectra of the solutions and solid films were recorded by a PerkinElmer LS55 fluorescence spectrometer. The plasma treatment on indium tin oxide (ITO) substrate was done in a Trion Sirns RIE Etch System. Spin-coating of the hole injection layer and the emitting layer was performed in a CEE 100 spin coater. The thickness of the spin-coating layers was measured by a Tencor P-10 Alpha step profiler. The thickness of thermally evaporated layers of electron injection/hole blocking layer and cathodes was monitored by a quartz crystal microbalance. The surface morphology was investigated by Digital Instruments Nanoscope IIIla atomic force microscopy (AFM). The current-voltage-luminance $(I-V-L)$ characteristics of the devices were recorded using a Keithley 2420 source meter, Keithley 2400 multimeter, and a silicon photodiode calibrated using a Minolta CS-200 Chromameter. Electroluminescent spectra of the devices were recorded with an Ocean Optics USB2000 miniature fiber optic spectrometer.

Device fabrication and characterization: The emissive layer was spincoated from solutions. Specifically, for undoped devices, the emitting materials were dissolved in toluene at concentration of $10 \mathrm{mg} \mathrm{mL}^{-1}$. For doped devices using PVK as host, Blu2 and PVK was dissolved in ethyl benzoate at a concentration of $5 \mathrm{mg} \mathrm{mL}^{-1}$. For devices using CBP as host, Blu2 and CBP was dissolved in chloroform at a concentration of $5 \mathrm{mg} \mathrm{mL}$. By controlling the weight ratio of the dopant and host in solution, we could control the concentration of emitter molecules in the host in the final solid devices. All these solutions are stirred in argon atmosphere overnight in darkness. The thickness of conductive ITO was about $110 \mathrm{~nm}$ with resistance of about $20 \Omega$ per square on glass that was used as a substrate for OLEDs. The refractive index of the ITO ranged from 1.94 to 2.04 in the wavelength range of $400 \mathrm{~nm}$ to $500 \mathrm{~nm}$, as specified by the vendor of the ITO used in this study. These values were similar to those in the literature. ${ }^{[49-51]}$ The refractive index of the glass was $\approx 1.5$ in the wavelength range, as specified by the vendor. ITO substrates were cleaned using detergent, deionized water, acetone, ethanol in an ultrasonic bath for $5 \mathrm{~min}$, consequently. To enhance the work function and improve the surface smoothness, the ITO surface was treated in argon-oxygen plasma for $10 \mathrm{~min}$ under RIE power of $100 \mathrm{~W}$ with oxygen flow of $30 \mathrm{sccm}$ and argon flow of $30 \mathrm{sccm}$ at pressure of 350 mTorr. PEDOT:PSS was spin-coated to form a hole injection layer of about $50 \mathrm{~nm}$ for all the devices, followed by drying at $120^{\circ} \mathrm{C}$ in air for $15 \mathrm{~min}$. The solution containing emitting materials was filtered through a $0.2 \mu \mathrm{m}$ poly (tetrafluoroethylene) (PTFE) filter right before spin-coating. By varying the spin rate, we can control the thickness of the emitting layer in the devices. After spin coating, the substrates with the spincoated layers were transferred to a chamber under vacuum of $1 \times 10^{-5} \mathrm{~Pa}$. A layer of $30 \mathrm{~nm}$ thick TPBI was deposited onto the surface of the emitting layer for electron injection and hole blocking. The cathode was composed of $0.5 \mathrm{~nm} \mathrm{LiF}$ and $150 \mathrm{~nm} \mathrm{Al}$, which were thermally deposited consecutively. All the measurements on devices were carried out in air at room temperature without encapsulation. The external quantum efficiencies were calculated using $I-V-L$ data and EL spectra of the devices, assuming Lambertian distribution of the EL emission. ${ }^{[52]}$

\section{Acknowledgements}

C.-G.Z. acknowledges support through a Rackham-MMPEI Energy Fellowship at the University of Michigan. Z.-K.C. would like to acknowledge the financial support from Institute of Materials Research \& Engineering (IMRE).

Received: October 13, 2010

Published online: January 18, 2011

[1] C. W. Tang, S. A. Vanslyke, Appl. Phys. Lett. 1987, 51, 913.

[2] Y. R. Sun, N. C. Giebink, H. Kanno, B. W. Ma, M. E. Thompson, S. R. Forrest, Nature 2006, 440, 908.

[3] S. Reineke, F. Lindner, G. Schwartz, N. Seidler, K. Walzer, B. Lussem, K. Leo, Nature 2009, 459, 234.

[4] B. E. A. Saleh, M. C. Teich, Fundamentals of Photonics, Wiley, New York 1991

[5] M. Baldo, M. Segal, Phys. Status Solidi A 2004, 201, 1205.

[6] R. H. Friend, R. W. Gymer, A. B. Holmes, J. H. Burroughes, R. N. Marks, C. Taliani, D. D. C. Bradley, D. A. Dos Santos, J. L. Bredas, M. Logdlund, W. R. Salaneck, Nature 1999, 397, 121.

[7] M. A. Baldo, M. E. Thompson, S. R. Forrest, Nature 2000, 403, 750.

[8] N. C. Giebink, S. R. Forrest, Phys. Rev. B 2008, 77, 235215.

[9] M. A. Baldo, C. Adachi, S. R. Forrest, Phys. Rev. B 2000, 62, 10967.

[10] W. Staroske, M. Pfeiffer, K. Leo, M. Hoffmann, Phys. Rev. Lett. 2007, 98, 197402. 
[11] Y. Cao, I. D. Parker, G. Yu, C. Zhang, A. J. Heeger, Nature 1999, 397, 414.

[12] M. Wohlgenannt, K. Tandon, S. Mazumdar, S. Ramasesha, Z. V. Vardeny, Nature 2001, 409, 494.

[13] Z. Shuai, D. Beljonne, R. J. Silbey, J. L. Bredas, Phys. Rev. Lett. 2000, 84, 131.

[14] S. Karabunarliev, E. R. Bittner, Phys. Rev. Lett. 2003, 90, 057402.

[15] Y. Wei, C. T. Chen, J. Am. Chem. Soc. 2007, 129, 7478

[16] K. Okumoto, H. Kanno, Y. Hamaa, H. Takahashi, K. Shibata, Appl. Phys. Lett. 2006, 89, 063504.

[17] H. Y. Chen, W. Y. Lam, J. D. Luo, Y. L. Ho, B. Z. Tang, D. B. Zhu, M. Wong, H. S. Kwok, Appl. Phys. Lett. 2002, 81, 574.

[18] S. L. Lin, L. H. Chan, R. H. Lee, M. Y. Yen, W. J. Kuo, C. T. Chen, R. J. Jeng, Adv. Mater. 2008, 20, 3947.

[19] S. K. Kim, B. Yang, Y. Ma, J. H. Lee, J. W. Park, J. Mater. Chem. 2008, 18, 3376.

[20] Y. Y. Lyu, J. Kwak, O. Kwon, S. H. Lee, D. Kim, C. Lee, K. Char, Adv. Mater. 2008, 20, 2720.

[21] C. H. Liao, M. T. Lee, C. H. Tsai, C. H. Chen, Appl. Phys. Lett. 2005, 86, 203507

[22] A. D. Becke, J. Chem. Phys. 1993, 98, 5648.

[23] Gaussian 03, Revision C.02, M. J. Frisch, G. W. Trucks, H. B. Schlegel, G. E. Scuseria, M. A. Robb, J. R. Cheeseman, J. A. Montgomery, Jr., T. Vreven, K. N. Kudin, J. C. Burant, J. M. Millam, S. S. Iyengar, J. Tomasi, V. Barone, B. Mennucci, M. Cossi, G. Scalmani, N. Rega, G. A. Petersson, H. Nakatsuji, M. Hada, M. Ehara, K. Toyota, R. Fukuda, J. Hasegawa, M. Ishida, T. Nakajima, Y. Honda, O. Kitao, H. Nakai, M. Klene, X. Li, J. E. Knox, H. P. Hratchian, J. B. Cross, V. Bakken, C. Adamo, J. Jaramillo, R. Gomperts, R. E. Stratmann O. Yazyev, A. J. Austin, R. Cammi, C. Pomelli, J. W. Ochterski, P. Y. Ayala, K. Morokuma, G. A. Voth, P. Salvador, J. J. Dannenberg, V. G. Zakrzewski, S. Dapprich, A. D. Daniels, M. C. Strain, O. Farkas, D. K. Malick, A. D. Rabuck, K. Raghavachari1, J. B. Foresman, J. V. Ortiz, Q. Cui, A. G. Baboul, S. Clifford, J. Cioslowski, B. B. Stefanov, G. Liu, A. Liashenko, P. Piskorz, I. Komaromi, R. L. Martin, D. J. Fox, T. Keith, M. A. Al-Laham, C. Y. Peng, A. Nanayakkara, M. Challacombe, P. M. W. Gill, B. Johnson, W. Chen, M. W. Wong, C. Gonzalez, J. A. Pople, Gaussian, Inc., Wallingford CT, 2004.

[24] R. A. Marcus, N. Sutin, Biochim. Biophys. Acta 1985, 811, 265.

[25] X. D. Yang, L. J. Wang, C. L. Wang, W. Long, Z. G. Shuai, Chem. Mater. 2008, 20, 3205.

[26] G. R. Hutchison, M. A. Ratner, T. J. Marks, J. Am. Chem. Soc. 2005, 127, 16866
[27] S. W. Yin, L. P. Chen, P. F. Xuan, K. Q. Chen, Z. Shuai, J. Phys. Chem B 2004, 108, 9608

[28] M. T. Sun, P. Kjellberg, W. J. D. Beenken, T. Pullerits, Chem. Phys. 2006, 327, 474.

[29] J. C. Sancho-Garcia, C. L. Foden, I. Grizzi, G. Greczynski, M. P. de Jong, W. R. Salaneck, J. L. Bredas, J. Cornil, J. Phys. Chem. B 2004, 108, 5594.

[30] L. H. Zhu, H. Q. Tang, Y. Harima, Y. Kunugi, K. Yamashita, J. Ohshita, A. Kunai, Thin Solid Films 2001, 396, 213.

[31] M. A. Lampert, P. Mark, Current Injection in Solids, Academic, New York, 1970.

[32] P. E. Burrows, Z. Shen, V. Bulovic, D. M. McCarty, S. R. Forrest, J. A. Cronin, M. E. Thompson, J. Appl. Phys. 1996, 79, 7991.

[33] Y. Yamamoto, R. E. Slusher, Phys. Today 1993, 46, 66.

[34] Y. Sato, H. Kanai, Mol. Cryst. Liq. Cryst. 1994, 252, 435.

[35] H. C. Cheung, Topics in Fluorescence Spectroscopy, Plenum, New York 1991. pp 129.

[36] S. C. Chang, G. He, F. C. Chen, T. F. Guo, Y. Yang, Appl. Phys. Lett. 2001, 79, 2088.

[37] Y. Kawamura, H. Sasabe, C. Adachi, Jpn. J. Appl. Phys., Part 1 2004, $43,7729$.

[38] Z. T. Liu, C. Y. Kwong, C. H. Cheung, A. B. Djurisic, Y. Chan, P. C. Chui, Synth. Met. 2005, 150, 159.

[39] B. W. D'Andrade, M. A. Baldo, C. Adachi, J. Brooks, M. E. Thompson, S. R. Forrest, Appl. Phys. Lett. 2001, 79, 1045.

[40] C. H. Chen, J. Shi, C. W. Tang, Macromol. Symp. 1998, 125, 1.

[41] M. A. Baldo, Z. G. Soos, S. R. Forrest, Chem. Phys. Lett. 2001, 347, 297.

[42] M. Pope, C. E. Swenberg, Electronic Processes in Organic Crystals, Oxford, New York 1982.

[43] D. Y. Kondakov, J. SID 2009, 17, 137.

[44] C. Ganzorig, M. Fujihira, Appl. Phys. Lett. 2002, 81, 3137.

[45] M. N. Kobrak, E. R. Bittner, Phys. Rev. B 2000, 62, 11473.

[46] L. P. Chen, L. Y. Zhu, Z. G. Shuai, J. Phys. Chem. A 2006, 110, 13349.

[47] H. Murata, C. D. Merritt, Z. H. Kafafi, IEEE J. Sel. Top. Quantum Electron. 1998, 4, 119.

[48] C. G. Zhen, Z. K. Chen, Q. D. Liu, Y. F. Dai, R. Y. C. Shin, S. Y. Chang, J. Kieffer, Adv. Mater. 2009, 21, 2425.

[49] J. Bartella, J. Schroeder, K. Witting, Appl. Surf. Sci. 2001, 179, 181.

[50] G. Franz, B. Lange, S. Sotier, J. Vac. Sci. Technol. A 2001, 19, 2514.

[51] K. Fehse, G. Schwartz, K. Walzer, K. Leo, J. Appl. Phys. 2007, 101,124509

[52] D. OBrien, A. Bleyer, D. G. Lidzey, D. D. C. Bradley, T. Tsutsui, J. Appl. Phys 1997, 82, 2662. 